\title{
In situ Synchrotron X-ray Tomographic Imaging of 3D Printed Materials During Uniaxial Loading.
}

Brian M. Patterson ${ }^{1}$, Nikolaus Cordes ${ }^{1}$, Kevin Henderson ${ }^{1}$, Robin Pacheco ${ }^{1}$, Matthew Herman ${ }^{1}$, James CE Mertens ${ }^{1}$, Xianghui Xiao ${ }^{2}$, Jason Williams ${ }^{3}$ and Nikhilesh Chawla ${ }^{3}$

1. Materials Science and Technology Division, Los Alamos National Laboratory, Los Alamos, NM, USA.

2. Argonne National Laboratory, Advanced Photon Source, Argonne, IL, USA.

3. Arizona State University, Tempe, AZ, USA.

The 3D printing of polymeric materials offer a wealth of possibilities, in that unique structures may be printed with geometries that are not possible with traditional molding, extrusion, casting, or machining techniques. Polymer structures may be optimized for weight, strength, or form, to improve their overall function. Mechanical testing indicates that print orientation as well as the use of recycled print material can affect the ultimate mechanical performance. Due to these problems, there are very few demonstrated high performance applications of 3D printed materials, especially polymers. In order to understand the adhesion between the printed layers as well as adhesion between the polymer and fillers and crack initiation, propagation, and ultimately failure, in situ analysis techniques are needed. To further complicate the analysis, these materials are typically hyper-elastic in nature. As such, experiments cannot be 'paused' during data collection as the material will continue to deform and respond to the applied stress.

Both laboratory based (on two size scales) and synchrotron based 3D tomographic imaging was used to study the deformation and fracture mechanics of 3D printed materials during uniaxial loading. Laboratory based 3D imaging using both micro-scale and nano-scale X-ray tomography captures the bending and buckling of materials during loading. Nano-scale imaging of lithographically printed lattices (Figure $1 \mathrm{left}$ ) shows the print quality of the polymer as well as deformation pathways. Microscale CT imaging provides a more global picture of the material response (Figure 1 right). Synchrotron X-ray tomographic imaging [1,2], using the fastest continuous 3D imaging yet demonstrated, four full 3D images were collected within one second as a loading rig was rotated at $2 \mathrm{~Hz}$, while applying a uniaxial load on 3D printed materials. Eighteen thousand $(18,000)$ radiographs were collected during this dynamic, five second event to be reconstructed into 20 tomograms. Dynamic stretching, cracking, failure, and elastic recovery were imaged in these hyper-elastic materials. Materials include glass bead filled polyamide 12 sintered powder (EOS material 3200 GF) (Figure 2) which were printed on an EOS Formiga P 110 printer. Simultaneously, the load response curve can be compared as a function of print conditions (Figure 2 right) and the mechanical response can be investigated using 3D digital volume correlation (Figure 3). The peak strain compares favorably for these small specimens as for full scale type IV tests.

\section{References:}

[1] Patterson BM et al, Journal of Material Science 51 (2016), p. 171-187. doi:DOI 10.1007/s10853-015-9355-8

[2] Cordes NL et al, Microscopy Today 23 (2015) (3) 

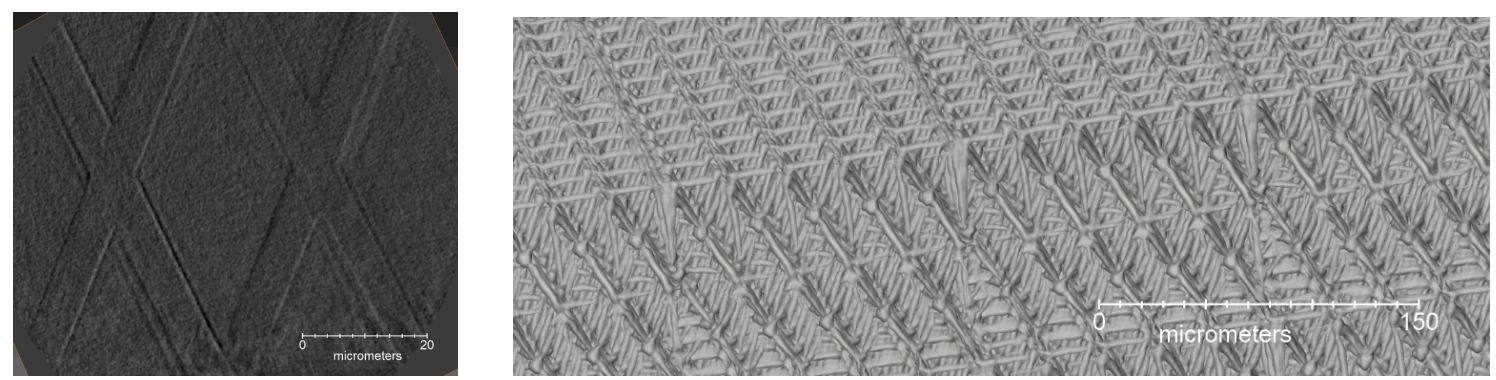

Figure 1. Left: Reconstructed slice using nano-scale (65 $\mu \mathrm{m}$ voxel) X-ray tomography of a 3D printed microlattice demonstrating print quality at this scale. Right: Micro-scale X-ray CT image of the same microlattice. This microlattice ligament diameter is just at the resolution limit of the CT instrument.
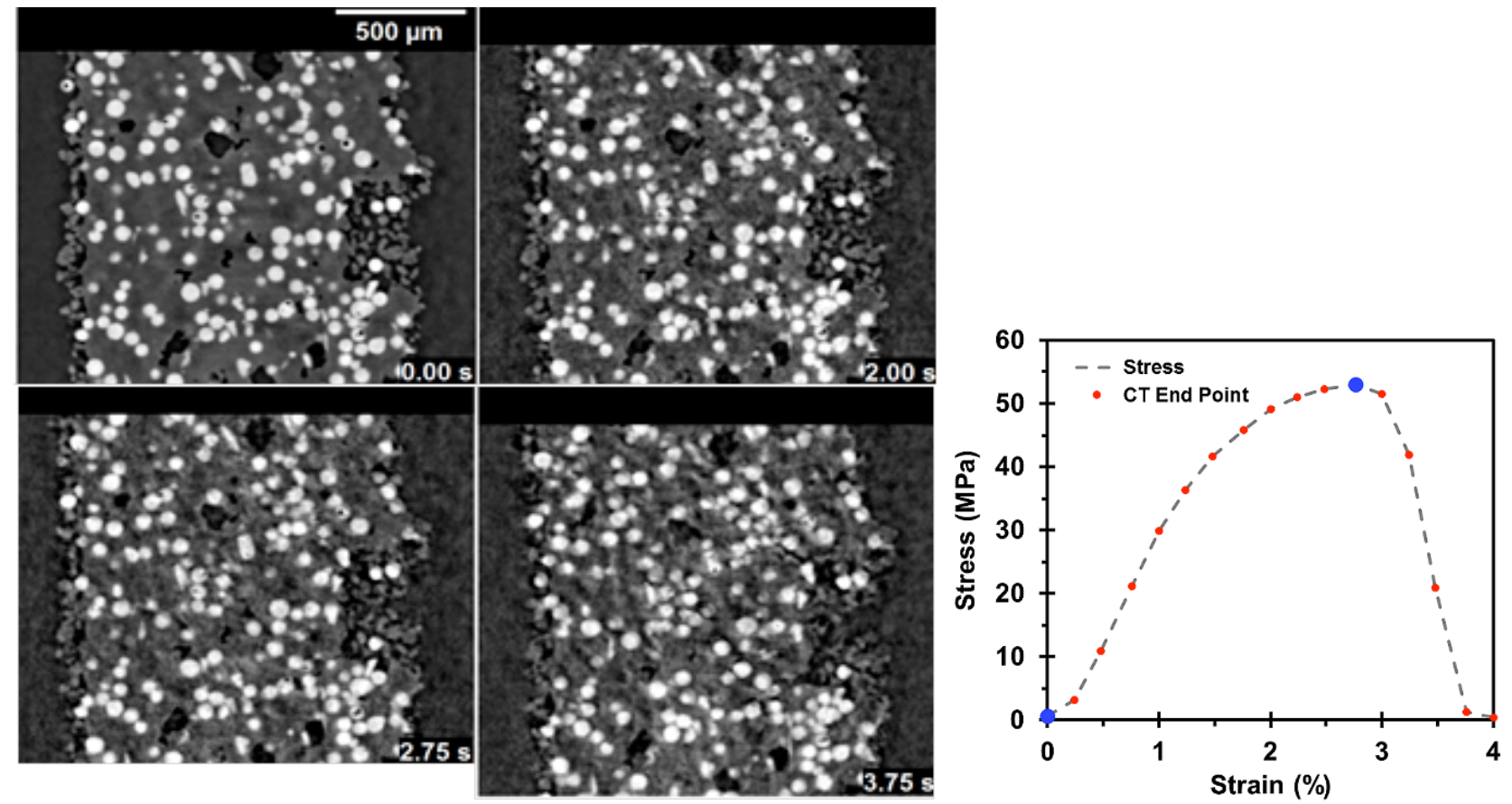

Figure 2: Reconstructed slices of the tensile loading of a glass bead filled 3D printed tensile specimen. The full 3D images were collected continuously and were 0.25 seconds apart. Only some are shown here. Separation of the polymer from the microbeads provides a weak pathway for the fracture of the specimen. Right: Entire gage-length-based strain calculation of the specimen collected during CT imaging.
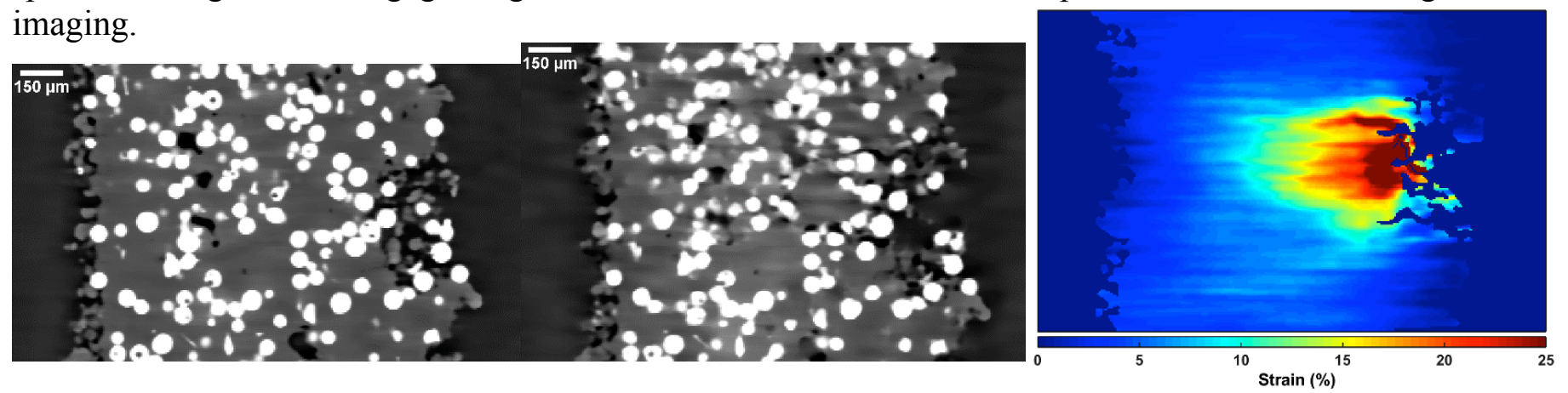

Figure 3: 'Identical' slice of the specimen shown in Figure 2 of CT\#0 and CT\#15 (4 seconds) processed for DVC. Loading direction is in the vertical direction and the notch is on the right. The DVC map of the strain in the vertical direction. 